\title{
Production and Hedging under State-Dependent Preferences and Background Risk*
}

\author{
Kit Pong Wong ${ }^{\dagger}$ \\ University of Hong Kong
}

July 2016

This paper examines the behavior of the competitive firm that possesses statedependent preferences and faces state-dependent price and background risk. While the background risk is neither hedgeable nor insurable, the firm has access to fairly priced futures and option contracts to hedge against the price risk. We show that the separation theorem holds in that the firm's optimal output level depends neither on the risk attitude of the firm nor on the incident to the underlying uncertainty. However, hedging does not always enhance production. We derive necessary and sufficient conditions under which the full-hedging theorem holds and thus options are not used. When these conditions are violated, we show that the firm optimally uses options to better cope with the complicated state-dependence structure between risk and preferences, thereby rendering options a hedging role over and above that of futures.

JEL classification: D21; D81; G13

Keywords: Futures; Options; Production; State-dependent preferences

\footnotetext{
*I would like to thank Udo Broll, Carl Chen (the editor), and two anonymous referees for their helpful comments and suggestions. The usual disclaimer applies.

${ }^{\dagger}$ Correspondence to: Kit Pong Wong, School of Economics and Finance, University of Hong Kong, Pokfulam Road, Hong Kong. Tel: +852-2859-1044, Fax: +852-2548-1152, e-mail: kpwong@econ.hku.hk
} 


\section{Introduction}

Since the seminal work of Holthausen (1979), there has been a large body of research on the hedging behavior of the competitive firm under price uncertainty à la Sandmo (1971). Two notable results emanate. First, the separation theorem states that the firm's optimal production decision depends neither on the risk attitude of the firm, nor on the incident of the underlying uncertainty when a futures market exists for hedging purposes. Second, the full-hedging theorem asserts that the firm should fully hedge against its exposure to the price risk if the futures market is unbiased. ${ }^{1}$ A corollary of the full-hedging theorem is that no other hedging instruments, options in particular, would play a hedging role that is over and above that of futures, rendering futures to be the most preferred hedging instrument (Battermann et al., 2000). ${ }^{2}$

The purposes of this paper are to examine the robustness of the separation and fullhedging theorems in general, and the hedging role of options in particular, under the premise that the competitive firm's preferences are state-dependent. There are legitimate reasons for considering state-dependent preferences. Karni et al. (1983) provide an axiomatization of expected utility maximizing behavior with subjective probabilities and state-dependent preferences. ${ }^{3}$ As convincingly argued by Briys and Schlesinger (1993), the state-dependentpreferences approach can be used to describe a reduced form of a more complex expected utility model that allows exogenous variations in wealth (Briys et al., 1993; Chavas, 1985) and/or relative prices (Adam-Müller, 2000; Broll and Wong, 2015). Following Broll and Eckwert (1998), Broll and Wong (2002), and Wong (2012a), we assume that the competitive firm possesses a bivariate state-dependent utility function that is defined over its profit and a state variable.

\footnotetext{
${ }^{1}$ The full-hedging theorem is analogous to a well-known result in the insurance literature that a risk-averse individual fully insures at an actuarially fair price (Mossin, 1968).

${ }^{2}$ Lapan et al. (1991) show that the competitive firm uses options only when it perceives the futures price and/or option premiums to be biased. Options as such act more like a speculative device rather than a hedging instrument.

${ }^{3}$ See Karni (1985) for a comprehensive introduction of the theory of state-dependent preferences.
} 
It goes without saying that multiple sources of uncertainty are a fact of life. We as such introduce the competitive firm other sources of risk that are aggregated into a single random variable. We refer to this random variable as background risk, which can be either additive or multiplicative in nature (Wong, 2013, 2014). While the firm has access to fairly priced futures and option contracts to hedge against the price risk, the background risk is neither hedgeable nor insurable. We allow the price risk (Broll and Wong, 2002; Wong, 2012a) and the background risk (Fei and Schlesinger, 2008; Wong, 2012b) to be state-dependent. For example, weather affects not only the supply of the commodity produced by the competitive firm, thereby the output price, but also the magnitude of losses when the firm's physical assets are on fire (Machnes, 1993; Wong, 1995, 1996). To this end, we consider a continuum of states of nature such that the joint probability distribution of the price and background risk is defined by the realization of the state variable. Following Fei and Schlesinger (2008) and Wong (2012b), we assume that the firm regards the price and background risk to be independent for a given realized state, but state-dependent when the realized state varies. Indeed, we show that the state-dependence structure between risk and preferences plays a pivotal role in shaping the firm's production and hedging decisions.

Since the competitive firm can always sell the last unit of its output through the futures contracts at the unbiased futures price, the firm must optimally produce at the point where the marginal cost of production is equated to the known marginal revenue, i.e., the unbiased futures price. The separation theorem as such is robust to the case of state-dependent preferences and in the presence of state-dependent price and background risk. We derive necessary and sufficient conditions under which banning the firm from hedging against the price risk has unambiguous effect on the firm's production decision. In contrast to the conventional wisdom, we construct a reasonable example to illustrate the plausible adverse effect of hedging on the firm's optimal output level. Finally, we derive necessary and sufficient conditions under which the full-hedging theorem holds and thus the firm does not use the option contracts for hedging purposes. When these conditions are violated, we show that the firm includes the option contracts in its optimal hedge position to better cope 
with the complicated state-dependence structure between risk and preferences. We as such provide a rationale for the hedging role of options, which is over and above that of futures, in the case of state-dependent preferences and in the presence of state-dependent price and background risk. ${ }^{4}$

This paper extends the models of Wong (2012a, 2012b) to incorporate both statedependent preferences and state-dependent price and background risk, where the background risk can be either additive or multiplicative. Like Wong (2012a, 2012b), we show that the separation theorem holds and there is a hedging role of options. Unlike Wong (2012a, 2012b), we show that hedging does not necessarily enhance the firm's production decision. We also derive general conditions under which the firm optimally opts for an unambiguous option position without imposing the additional assumption of prudence in the sense of Kimball $(1990,1993)$.

The rest of this paper is organized as follows. Section 2 delineates the model of the competitive firm that possesses state-dependent preferences and faces state-dependent price and background risk, where the background risk can be either additive or multiplicative. The firm has access to fairly priced futures and option contracts to hedge against the price risk. Section 3 characterizes the firm's optimal production decision and shows the plausible adverse effect of hedging on output. Section 4 derives the firm's optimal hedge position and shows the hedging role of options. The final section concludes.

\section{The model}

Consider the competitive firm of Sandmo (1971) that operates in one period with two dates, 0 and 1 . The firm possesses a bivariate state-dependent utility function, $U(\Pi, S)$, defined over its profit, $\Pi$, at date 1 , and the realization of a random variable, $\tilde{S}$, that maps

\footnotetext{
${ }^{4}$ Machnes (1992) and Wong (2002) restrict the competitive firm to use options only for hedging purposes, thereby failing to show the hedging role of options.
} 
states of nature to real numbers. Let $H(S)$ be the cumulative distribution function (CDF) of $\tilde{S}$ over support $[\underline{S}, \bar{S}]$, where $\underline{S}<\bar{S}$. The firm is risk averse in that $U_{\Pi}(\Pi, S)>0$ and $U_{\Pi \Pi}(\Pi, S)<0$ for all $\Pi \geq 0$ and $S \in[\underline{S}, \bar{S}]$, where subscripts indicate partial derivatives.

To begin, the firm produces a single commodity according to a deterministic cost function, $C(Q)$, where $Q \geq 0$ is the output level chosen by the firm at date 0 , and $C(Q)$ is compounded to date 1 . The firm's production technology exhibits decreasing returns to scale so that the cost function, $C(Q)$, satisfies that $C(0)=C^{\prime}(0)=0$, and $C^{\prime}(Q)>0$ and $C^{\prime \prime}(Q)>0$ for all $Q>0$. At date 1, the firm sells the entirety of its output level, $Q$, at the then prevailing per-unit price, $\tilde{P}$, which is a positive random variable not known ex ante. ${ }^{5}$ Conditioned on the realized state, $S, \tilde{P}$ is distributed according to a $\mathrm{CDF}, F(P \mid S)$, over support $[\underline{P}, \bar{P}]$, where $0<\underline{P}<\bar{P}$. Let $\mathrm{E}_{F}(\cdot \mid S)$ be the expectation operator with respect to the CDF, $F(P \mid S)$.

Besides the price risk, $\tilde{P}$, the firm faces other sources of risk that are aggregated into a single random variable, $\tilde{Z}$. We refer to $\tilde{Z}$ as the background risk, and assume that it is either additive or multiplicative in nature. Conditioned on the realized state, $S, \tilde{Z}$ is distributed according to a CDF, $G(Z \mid S)$, over support $[\underline{Z}, \bar{Z}]$, where $\underline{Z}<0<\bar{Z}$. Let $\mathrm{E}_{G}(\cdot \mid S)$ be the expectation operator with respect to the CDF, $G(Z \mid S)$. We normalize the unconditional expected value of $\tilde{Z}$ to zero, i.e.,

$$
\int_{\underline{S}}^{\bar{S}} \mathrm{E}_{G}(\tilde{Z} \mid S) \mathrm{d} H(S)=0 .
$$

For the sake of tractability, we follow Wong (2012b) to assume that the price risk, $\tilde{P}$, is independent of the background risk, $\tilde{Z}$, conditional on the realized state, $S$. However, as the realized state varies, $\tilde{P}$ and $\tilde{Z}$ are deemed to be state-dependent. To see this, we calculate the covariance between $A(\tilde{P})$ and $B(\tilde{Z})$ :

$$
\int_{\underline{S}}^{\bar{S}} \int_{\underline{P}}^{\bar{P}} \int_{\underline{Z}}^{\bar{Z}}\left\{A(P)-\int_{\underline{S}}^{\bar{S}} \mathrm{E}_{F}[A(\tilde{P}) \mid S] \mathrm{d} H(S)\right\}
$$

\footnotetext{
${ }^{5}$ Throughout the paper, random variables have a tilde $(\sim)$ while their realizations do not.
} 


$$
\begin{gathered}
\times\left\{B(Z)-\int_{\underline{S}}^{\bar{S}} \mathrm{E}_{G}[B(\tilde{Z}) \mid S] \mathrm{d} H(S)\right\} \mathrm{d} F(P \mid S) \mathrm{d} G(Z \mid S) \mathrm{d} H(S) \\
=\int_{\underline{S}}^{\bar{S}}\left\{\mathrm{E}_{F}[A(\tilde{P}) \mid S]-\int_{\underline{S}}^{\bar{S}} \mathrm{E}_{F}[A(\tilde{P}) \mid S] \mathrm{d} H(S)\right\} \\
\times\left\{\mathrm{E}_{G}[B(\tilde{Z}) \mid S]-\int_{\underline{S}}^{\bar{S}} \mathrm{E}_{G}[B(\tilde{Z}) \mid S] \mathrm{d} H(S)\right\} \mathrm{d} H(S) \\
=\operatorname{Cov}_{H}\left\{\mathrm{E}_{F}[A(\tilde{P}) \mid \tilde{S}], \mathrm{E}_{G}[B(\tilde{Z}) \mid \tilde{S}]\right\},
\end{gathered}
$$

where $A(\cdot)$ and $B(\cdot)$ are two arbitrarily chosen functions, and $\operatorname{Cov}_{H}(\cdot, \cdot)$ is the covariance operator with respect to the $\mathrm{CDF}, H(S)$. It follows from Eq. (2) that $A(\tilde{P})$ and $B(\tilde{Z})$ are positively (negatively) state-dependent if, and only if, $\operatorname{Cov}_{H}\left\{\mathrm{E}_{F}[A(\tilde{P}) \mid \tilde{S}], \mathrm{E}_{G}[B(\tilde{Z}) \mid \tilde{S}]\right\}>(<) 0$. For example, this is the case when an increase in $S$ affects $\mathrm{E}_{F}[A(\tilde{P}) \mid S]$ and $\mathrm{E}_{G}[B(\tilde{Z}) \mid S]$ in the same direction (opposite directions).

While the background risk, $\tilde{Z}$, is neither hedgeable nor insurable, the firm can hedge against the price risk, $\tilde{P}$, by trading infinitely divisible futures and put option contracts at date 0 , each of which calls for delivery of one unit of the commodity at date $1 .{ }^{6}$ The futures price is predetermined at $P^{f}$ at date 0 , where $\underline{P}<P^{f}<\bar{P}$. The put option contracts have a single strike price, $K$, at which the option holders have the right but not the obligation to sell one unit of the commodity per contract at date 1 , where $\underline{P}<K<\bar{P}$. The option premium per contract is exogenously fixed at $\Phi>0$, which is compounded to date 1 . To focus on the firm's pure hedging motive, we assume that the futures and put option contracts are fairly priced in that

$$
P^{f}=\int_{\underline{S}}^{\bar{S}} \mathrm{E}_{F}(\tilde{P} \mid S) \mathrm{d} H(S)
$$

and

$$
\Phi=\int_{\underline{S}}^{\bar{S}} \mathrm{E}_{F}[\max (K-\tilde{P}, 0) \mid S] \mathrm{d} H(S) .
$$

\footnotetext{
${ }^{6}$ Because of the put-call parity, payoffs of any combinations of futures, calls, and puts can be replicated by any two of these three financial contracts, rendering one of them to be redundant. Restricting the firm to use only futures and put option contracts is, therefore, without any loss of generality.
} 
Eqs. (3) and (4) imply that $P^{f}$ and $\Phi$ are set equal to the unconditional expected value of $\tilde{P}$ and that of $\max (K-\tilde{P}, 0)$, respectively.

Let the pair, $(X, Y)$, denote the firm's hedge position, where $X$ is the number of the futures contracts sold (purchased if negative), and $Y$ is the numbers of the put option contracts purchased (sold if negative) by the firm at date 0 . The firm's profit at date 1 is given by

$$
\begin{aligned}
\Pi(\tilde{P}, \tilde{Z})= & (1+\theta \tilde{Z})\left\{\tilde{P} Q-C(Q)+\left(P^{f}-\tilde{P}\right) X+[\max (K-\tilde{P}, 0)-\Phi] Y\right\} \\
& +(1-\theta) \tilde{Z}
\end{aligned}
$$

where $\theta=0$ or 1 , depending on whether the background risk is additive or multiplicative in nature, respectively. ${ }^{7}$ We refer to the firm's futures position, $X$, as an under-hedge, a full-hedge, or an over-hedge, depending on whether $X$ is smaller than, equal to, or greater than the output level, $Q$, respectively. We refer to the firm's put option position, $Y$, as a long or short position, depending on whether $Y$ is positive or negative, respectively.

The firm's ex-ante decision problem is to choose an output level, $Q \geq 0$, and a hedge position, $(X, Y)$, at date 0 so as to maximize the expected utility of its profit at date 1 :

$$
\max _{Q \geq 0, X, Y} \int_{\underline{S}}^{\bar{S}} \int_{\underline{P}}^{\bar{P}} \int_{\underline{Z}}^{\bar{Z}} U[\Pi(P, Z), S] \mathrm{d} F(P \mid S) \mathrm{d} G(Z \mid S) \mathrm{d} H(S),
$$

where $\Pi(P, Z)$ is given by Eq. (5). The first-order conditions for program (6) are given by

$$
\begin{aligned}
& \int_{\underline{S}}^{\bar{S}} \int_{\underline{P}}^{\bar{P}} \int_{\underline{Z}}^{\bar{Z}} U_{\Pi}\left[\Pi^{*}(P, Z), S\right](1+\theta Z)\left[P-C^{\prime}\left(Q^{*}\right)\right] \mathrm{d} F(P \mid S) \mathrm{d} G(Z \mid S) \mathrm{d} H(S)=0, \\
& \int_{\underline{S}}^{\bar{S}} \int_{\underline{P}}^{\bar{P}} \int_{\underline{Z}}^{\bar{Z}} U_{\Pi}\left[\Pi^{*}(P, Z), S\right](1+\theta Z)\left(P^{f}-P\right) \mathrm{d} F(P \mid S) \mathrm{d} G(Z \mid S) \mathrm{d} H(S)=0,
\end{aligned}
$$

and

$$
\int_{\underline{S}}^{\bar{S}} \int_{\underline{P}}^{\bar{P}} \int_{\underline{Z}}^{\bar{Z}} U_{\Pi}\left[\Pi^{*}(P, Z), S\right](1+\theta Z)
$$

\footnotetext{
${ }^{7}$ When $\theta=1$, we assume that $1+\tilde{Z}>0$.
} 


$$
\times[\max (K-P, 0)-\Phi] \mathrm{d} F(P \mid S) \mathrm{d} G(Z \mid S) \mathrm{d} H(S)=0,
$$

where an asterisk $\left({ }^{*}\right)$ indicates an optimal level. The second-order conditions for program (6) are satisfied given the assumed properties of $U(\Pi, S)$ and $C(Q)$.

\section{Optimal production decision}

In this section, we examine the firm's optimal production decision. To this end, we add Eq. (7) to Eq. (8) to yields

$$
\int_{\underline{S}}^{\bar{S}} \int_{\underline{P}}^{\bar{P}} \int_{\underline{Z}}^{\bar{Z}} U_{\Pi}\left[\Pi^{*}(P, Z), S\right](1+\theta Z)\left[P^{f}-C^{\prime}\left(Q^{*}\right)\right] \mathrm{d} F(P \mid S) \mathrm{d} G(Z \mid S) \mathrm{d} H(S)=0 .
$$

It follows that Eq. (10) reduces to $C^{\prime}\left(Q^{*}\right)=P^{f}$, thereby invoking our first proposition.

Proposition 1. Given that the competitive firm can hedge against the price risk, $\tilde{P}$, by trading the fairly priced futures and put option contracts, the firm's optimal output level, $Q^{*}$, is the one that equates the marginal cost of production, $C^{\prime}\left(Q^{*}\right)$, to the unbiased futures price, $P^{f}$.

The intuition for Proposition 1 is as follows. Since the firm can always sell the last unit of its output through the futures contracts at the unbiased futures price, $P^{f}$, the usual optimality condition applies in that the marginal cost of production, $C^{\prime}\left(Q^{*}\right)$, must be equated to the known marginal revenue, $P^{f}$, which determines the optimal output level, $Q^{*}$. An immediate implication of Proposition 1 is that the firm's optimal production decision depends neither on the firm's state-dependent preferences nor on the incident to the multiple sources of uncertainty. Proposition 1 as such extends the separation theorem to the case of state-dependent preferences and in the presence of state-dependent price and background risk. It is worth pointing out that Proposition 1 remains valid when the state-dependent background risk is absent, i.e., $\tilde{Z} \equiv 0$. 
It is of interest to examine the effect of hedging on the firm's optimal production decision. To this end, we consider a benchmark case wherein the firm is banned from trading the futures and put option contracts, i.e., $X \equiv 0$ and $Y \equiv 0$. The first-order condition for program (6) becomes

$$
\int_{\underline{S}}^{\bar{S}} \int_{\underline{P}}^{\bar{P}} \int_{\underline{Z}}^{\bar{Z}} U_{\Pi}\left[\Pi_{0}^{\circ}(P, Z), S\right](1+\theta Z)\left[P-C^{\prime}\left(Q^{\circ}\right)\right] \mathrm{d} F(P \mid S) \mathrm{d} G(Z \mid S) \mathrm{d} H(S)=0,
$$

where $\Pi_{0}^{\circ}(P, Z)=(1+\theta Z)\left[P Q^{\circ}-C\left(Q^{\circ}\right)\right]+(1-\theta) Z$, and $Q^{\circ}$ is the optimal output level in this benchmark case.

To compare $Q^{\circ}$ with $Q^{*}$, we evaluate the left-hand side of Eq. (11) at $Q^{*}$ to yield

$$
\begin{aligned}
& \int_{\underline{S}}^{\bar{S}} \int_{\underline{P}}^{\bar{P}} \int_{\underline{Z}}^{\bar{Z}} U_{\Pi}\left[\Pi_{0}^{*}(P, Z), S\right](1+\theta Z)\left[P-C^{\prime}\left(Q^{*}\right)\right] \mathrm{d} F(P \mid S) \mathrm{d} G(Z \mid S) \mathrm{d} H(S) \\
& =\int_{\underline{S}}^{\bar{S}} \int_{\underline{P}}^{\bar{P}} \int_{\underline{Z}}^{\bar{Z}} U_{\Pi}\left[\Pi_{0}^{*}(P, Z), S\right](1+\theta Z)\left(P-P^{f}\right) \mathrm{d} F(P \mid S) \mathrm{d} G(Z \mid S) \mathrm{d} H(S),
\end{aligned}
$$

where $\Pi_{0}^{*}(P, Z)=(1+\theta Z)\left[P Q^{*}-C\left(Q^{*}\right)\right]+(1-\theta) Z$, and the equality follows from Proposition 1 that $C^{\prime}\left(Q^{*}\right)=P^{f}$. Eq. (11) and the second-order conditions for program (6) then imply that $Q^{\circ}<(>) Q^{*}$ if, and only if, the right-hand side of Eq. (12) is negative (positive), thereby invoking the following proposition.

Proposition 2. Banning the competitive firm from hedging against the price risk, $\tilde{P}$, by trading the fairly priced futures and put option contracts induces the firm to optimally produce less (more), i.e., $Q^{\circ}<(>) Q^{*}$, if, and only if, the following condition holds:

$$
P^{f}>(<) \frac{\int_{\underline{S}}^{\bar{S}} \int_{\underline{P}}^{\bar{P}} \mathrm{E}_{G}\left\{U_{\Pi}\left[\Pi_{0}^{*}(P, \tilde{Z}), S\right](1+\theta \tilde{Z}) \mid S\right\} P \mathrm{~d} F(P \mid S) \mathrm{d} H(S)}{\int_{\underline{S}}^{\bar{S}} \int_{\underline{P}}^{\bar{P}} \mathrm{E}_{G}\left\{U_{\Pi}\left[\Pi_{0}^{*}(P, \tilde{Z}), S\right](1+\theta \tilde{Z}) \mid S\right\} \mathrm{d} F(P \mid S) \mathrm{d} H(S)},
$$

where $\Pi_{0}^{*}(P, Z)=(1+\theta Z)\left[P Q^{*}-C\left(Q^{*}\right)\right]+(1-\theta) Z$.

To see the intuition for Proposition 2, we define the following function:

$$
\hat{F}(P \mid S)=\int_{\underline{P}}^{P} \frac{\mathrm{E}_{G}\left\{U_{\Pi}\left[\Pi_{0}^{*}(p, \tilde{Z}), S\right](1+\theta \tilde{Z}) \mid S\right\}}{\int_{\underline{P}}^{\bar{P}} \mathrm{E}_{G}\left\{U_{\Pi}\left[\Pi_{0}^{*}(P, \tilde{Z}), S\right](1+\theta \tilde{Z}) \mid S\right\} \mathrm{d} F(P \mid S)} \mathrm{d} F(p \mid S),
$$


for all $P \in[\underline{P}, \bar{P}]$ and $S \in[\underline{S}, \bar{S}]$. It is evident from Eq. (14) that $\hat{F}(\underline{P} \mid S)=0, \hat{F}(\bar{P} \mid S)=1$, and $\partial \hat{F}(P \mid S) / \partial P>0$ for all $P \in[\underline{P}, \bar{P}]$ so that we can interpret $\hat{F}(P \mid S)$ as a CDF of $\tilde{P}$ conditional on the realized state, $S$. Likewise, we define the following function:

$$
\hat{H}(S)=\int_{\underline{S}}^{S} \frac{\int_{\underline{P}}^{\bar{P}} \mathrm{E}_{G}\left\{U_{\Pi}\left[\Pi_{0}^{*}(P, \tilde{Z}), s\right](1+\theta \tilde{Z}) \mid s\right\} \mathrm{d} F(P \mid s)}{\int_{\underline{S}}^{\bar{S}} \int_{\underline{P}}^{\bar{P}} \mathrm{E}_{G}\left\{U_{\Pi}\left[\Pi_{0}^{*}(P, \tilde{Z}), S\right](1+\theta \tilde{Z}) \mid S\right\} \mathrm{d} F(P \mid S) \mathrm{d} H(S)} \mathrm{d} H(s),
$$

for all $S \in[\underline{S}, \bar{S}]$. It is evident from Eq. (15) that $\hat{H}(\underline{S})=0, \hat{H}(\bar{S})=1$, and $\hat{H}^{\prime}(S)>0$ for all $S \in[\underline{S}, \bar{S}]$ so that we can interpret $\hat{H}(S)$ as a CDF of $\tilde{S}$. Using Eqs. (14) and (15), we can write condition (13) as

$$
P^{f}>(<) \int_{\underline{S}}^{\bar{S}} \mathrm{E}_{\hat{F}}(\tilde{P} \mid S) \mathrm{d} \hat{H}(S)
$$

where $\mathrm{E}_{\hat{F}}(\cdot \mid S)$ is the expectation operator with respect to the $\mathrm{CDF}, \hat{F}(P \mid S)$. We can interpret the right-hand side of condition (16) as the certainty equivalent spot price at date 1 , taking the firm's state-dependent preferences and the underlying multiple sources of uncertainty into account. When trading the fairly priced futures and put option contracts is allowed, we know from Proposition 1 that the firm produces the optimal output level, $Q^{*}$, at which the marginal cost of production, $C^{\prime}\left(Q^{*}\right)$, is equal to the unbiased futures price, $P^{f}$. Condition (16) ensures that $C^{\prime}\left(Q^{*}\right)$ is above (below) the certainty equivalent spot price at date 1 when the firm is banned from hedging, thereby rendering the firm to lower (raise) its optimal output level, i.e., $Q^{\circ}<(>) Q^{*}$.

To illustrate the plausible adverse effect of hedging on the firm's optimal output level, we employ the parametric example of Broll and Eckwert (1998) in which the firm's statedependent utility function is given by $U(\Pi, S)=S \Pi-\gamma \Pi^{2} / 2$, where $\gamma$ is a positive constant such that $U_{\Pi}(\Pi, S)=S-\gamma \Pi>0$ for all relevant values of $\Pi$. Suppose that an increase in the realized state, $S$, induces a decrease in the price risk, $\tilde{P}$, by the first-order stochastic dominance so that $\partial \mathrm{E}_{F}(\tilde{P} \mid S) / \partial S>0$. Furthermore, suppose that an increase in $S$ induces a mean-preserving-spread increase in the background risk, $\tilde{Z}$, in the sense of Rothschild and Stiglitz (1970) so that $\partial \mathrm{E}_{G}(\tilde{Z} \mid S) / \partial S=0$ and $\partial \mathrm{E}_{G}\left(\tilde{Z}^{2} \mid S\right) / \partial S>0$. To ensure $Q^{\circ}>Q^{*}$, 
condition (13) becomes

$$
\begin{aligned}
\gamma<\operatorname{Cov}_{H}\left[\tilde{S}, \mathrm{E}_{F}(\tilde{P} \mid \tilde{S})\right] /\{ & \operatorname{Var}(\tilde{P}) Q^{*}+\theta Q^{*} \int_{\underline{S}}^{\bar{S}} \mathrm{E}_{F}\left[\left(\tilde{P}-P^{f}\right)^{2} \mid S\right] \mathrm{E}_{G}\left(\tilde{Z}^{2} \mid S\right) \mathrm{d} H(S) \\
& \left.+\theta \operatorname{Cov}_{H}\left[\mathrm{E}_{F}(\tilde{P} \mid \tilde{S}), \mathrm{E}_{G}\left(\tilde{Z}^{2} \mid \tilde{S}\right)\right]\left[P^{f} Q^{*}-C\left(Q^{*}\right)\right]\right\},
\end{aligned}
$$

where $\operatorname{Var}(\tilde{P})$ is the variance of $\tilde{P}$. Since $Q^{*}$ solves $C^{\prime}\left(Q^{*}\right)=P^{f}$, the right-hand side of inequality (17), which is positive, does not depend on $\gamma$. Hence, when $\gamma$ is sufficiently small in that inequality (17) holds, hedging with the fairly priced futures and put option contracts adversely affects the firm's production decision. When the state-dependent background risk is absence, i.e., $\tilde{Z} \equiv 0$, the right-hand side of inequality (17) remains the same if $\theta=0$ and becomes larger if $\theta=1$, thereby making the adverse effect of hedging on output not less likely in this example.

\section{Optimal hedging decision}

In this section, we examine the firm's optimal hedging decision in general and the hedging role of options in particular. To this end, we reformulate program (6) as a two-stage optimization problem by fixing the firm's output level at $Q^{*}$. In the first stage, we derive the firm's demand for the unbiased futures contracts, $X(Y)$, where $X(Y)$ maximizes the objective function of program (6) for a fixed put option position, $Y$ :

$$
\int_{\underline{S}}^{\bar{S}} \int_{\underline{P}}^{\bar{P}} \int_{\underline{Z}}^{\bar{Z}} U_{\Pi}[\Pi(P, Z \mid Y), S](1+\theta Z)\left(P^{f}-P\right) \mathrm{d} F(P \mid S) \mathrm{d} G(Z \mid S) \mathrm{d} H(S)=0
$$

where $\Pi(P, Z \mid Y)=(1+\theta Z)\left\{P Q^{*}-C\left(Q^{*}\right)+\left(P^{f}-P\right) X(Y)+[\max (K-P, 0)-\Phi] Y\right\}+(1-$ $\theta) Z$. In the second stage, we derive the firm's optimal put option position, $Y^{*}$, where $Y^{*}$ maximizes the objective function of program (6) with $X$ replaced by $X(Y)$. The complete solution to program (6) is, therefore, given by $Y^{*}$ and $X^{*}=X\left(Y^{*}\right){ }^{8}$

\footnotetext{
${ }^{8}$ Since the firm is the sole decision maker, the order of the two stages is inconsequential to the firm's optimal hedge position, $\left(X^{*}, Y^{*}\right)$.
} 
Differentiating the objective function of program (6) with $X=X(Y)$ with respect to $Y$, and evaluating the resulting derivative at $Y=0$ yields

$$
\begin{gathered}
\left.\frac{\partial}{\partial Y} \int_{\underline{S}}^{\bar{S}} \int_{\underline{P}}^{\bar{P}} \int_{\underline{Z}}^{\bar{Z}} U[\Pi(P, Z), S] \mathrm{d} F(P \mid S) \mathrm{d} G(Z \mid S) \mathrm{d} H(S)\right|_{X=X(0), Y=0} \\
=\int_{\underline{S}}^{\bar{S}} \int_{\underline{P}}^{\bar{P}} \int_{\underline{Z}}^{\bar{Z}} U_{\Pi}[\Pi(P, Z \mid 0), S](1+\theta Z) \\
\quad \times[\max (K-\tilde{P}, 0)-\Phi] \mathrm{d} F(P \mid S) \mathrm{d} G(Z \mid S) \mathrm{d} H(S),
\end{gathered}
$$

where $\Pi(P, Z \mid 0)=(1+\theta Z)\left[P Q^{*}-C\left(Q^{*}\right)+\left(P^{f}-P\right) X(0)\right]+(1-\theta) Z$. If the right-hand side of Eq. (19) is positive (negative), it follows from Eq. (9) and the second-order conditions for program (6) that $Y^{*}>(<) 0$.

The full-hedging theorem applies when the firm optimally adopts a full-hedge, i.e., $X(0)=Q^{*}$, and uses no options, i.e., $Y^{*}=0$. Using Eqs. (18) and (19), this is the case if, and only if, the following two equations hold simultaneously:

$$
\int_{\underline{S}}^{\bar{S}} \mathrm{E}_{G}\left\{U_{\Pi}\left[\Pi_{0}^{*}\left(P^{f}, \tilde{Z}\right), S\right](1+\theta \tilde{Z}) \mid S\right\}\left[P^{f}-\mathrm{E}_{F}(\tilde{P} \mid S)\right] \mathrm{d} H(S)=0,
$$

and

$$
\int_{\underline{S}}^{\bar{S}} \mathrm{E}_{G}\left\{U_{\Pi}\left[\Pi_{0}^{*}\left(P^{f}, \tilde{Z}\right), S\right](1+\theta \tilde{Z}) \mid S\right\}\left\{\mathrm{E}_{F}[\max (K-\tilde{P}, 0) \mid S]-\Phi\right\} \mathrm{d} H(S)=0,
$$

where $\Pi_{0}^{*}\left(P^{f}, Z\right)=(1+\theta Z)\left[P^{f} Q^{*}-C\left(Q^{*}\right)\right]+(1-\theta) Z$. The following proposition follows immediately from Eqs. (20) and (21).

Proposition 3. Given that the competitive firm can hedge against the price risk, $\tilde{P}$, by trading the fairly priced futures and put option contracts, the firm optimally opts for a fullhedge, i.e., $X^{*}=Q^{*}$, and uses no options, i.e., $Y^{*}=0$, if, and only if, the following two equations hold simultaneously:

$$
\operatorname{Cov}_{H}\left\{\mathrm{E}_{G}\left\{U_{\Pi}\left[\Pi_{0}^{*}\left(P^{f}, \tilde{Z}\right), \tilde{S}\right](1+\theta \tilde{Z}) \mid \tilde{S}\right\}, \mathrm{E}_{F}(\tilde{P} \mid \tilde{S})\right\}=0
$$


and

$$
\operatorname{Cov}_{H}\left\{\mathrm{E}_{G}\left\{U_{\Pi}\left[\Pi_{0}^{*}\left(P^{f}, \tilde{Z}\right), \tilde{S}\right](1+\theta \tilde{Z}) \mid \tilde{S}\right\}, \mathrm{E}_{F}[\max (K-\tilde{P}, 0) \mid \tilde{S}]\right\}=0
$$

where $\Pi_{0}^{*}\left(P^{f}, Z\right)=(1+\theta Z)\left[P^{f} Q^{*}-C\left(Q^{*}\right)\right]+(1-\theta) Z$.

Proof. Using the property of the covariance operator, $\operatorname{Cov}_{H}(\cdot, \cdot)$, we can substitute Eq. (3) into Eq. (20) to yield Eq. (22). ${ }^{9}$ Likewise, we can substitute Eq. (4) into Eq. (21) to yield Eq. (23).

Proposition 3 provides the necessary and sufficient conditions under which the fullhedging theorem applies to the firm's hedging decisions. To show that these conditions may hold, we consider a special case wherein the firm's state-dependent utility function is given by $U(\Pi, S)=S+\Pi-\gamma \Pi^{2} / 2$, where $\gamma$ is a positive constant such that $U_{\Pi}(\Pi, S)=1-\gamma \Pi>0$ for all relevant values of $\Pi$. In this case, Eqs. (22) and (23) reduce to

$$
\operatorname{Cov}_{H}\left[\theta \mathrm{E}_{G}\left(\tilde{Z}^{2} \mid \tilde{S}\right)+(1+\theta) \mathrm{E}_{G}(\tilde{Z} \mid \tilde{S}), \mathrm{E}_{F}(\tilde{P} \mid \tilde{S})\right]=0
$$

and

$$
\operatorname{Cov}_{H}\left\{\theta \mathrm{E}_{G}\left(\tilde{Z}^{2} \mid \tilde{S}\right)+(1+\theta) \mathrm{E}_{G}(\tilde{Z} \mid \tilde{S}), \mathrm{E}_{F}[\max (K-\tilde{P}, 0) \mid \tilde{S}]\right\}=0
$$

respectively. Suppose that the background risk, $\tilde{Z}$, preserves its expected value and variance as the realized state, $S$, varies so that $\mathrm{E}_{G}(\tilde{Z} \mid S)=0$ and $\mathrm{E}_{G}\left(\tilde{Z}^{2} \mid S\right)=\sigma_{Z}^{2}$ for all $S \in[\underline{S}, \bar{S}]$, where $\sigma_{Z}^{2}>0$ is the variance of $\tilde{Z} .^{10}$ In this example, it is evident that Eqs. (24) and (25) hold simultaneously, thereby rendering the validity of the full-hedging theorem.

Of course, the above example assumes that the state variable, $S$, shifts the statedependent utility function, $U(\Pi, S)$, in a parallel fashion, which is unduly restrictive. This

\footnotetext{
${ }^{9}$ For any two random variables, $\tilde{X}$ and $\tilde{Y}$, we have $\operatorname{Cov}_{H}(\tilde{X}, \tilde{Y})=\mathrm{E}_{H}(\tilde{X} \tilde{Y})-\mathrm{E}_{H}(\tilde{X}) \mathrm{E}_{H}(\tilde{Y})$.

${ }^{10}$ For example, this is the case when the background risk, $\tilde{Z}$, exhibits an increase in downside risk in the sense of Menezes et al. (1980) as the realized state, $S$, increases.
} 
suggests that Eqs. (22) and (23) are unlikely to hold simultaneously. In the following proposition, we derive necessary and sufficient conditions under which $Y^{*}>(<) 0$.

Proposition 4. Given that the competitive firm can hedge against the price risk, $\tilde{P}$, by trading the fairly priced futures and put option contracts, and that the expected price risk, $\mathrm{E}_{F}(\tilde{P} \mid S)$, is preserved as the realized state, $S$, varies, the firm optimally opts for a long (short) put option position, i.e., $Y^{*}>(<) 0$, if, and only if, the following condition holds:

$$
\operatorname{Cov}_{H}\left\{\mathrm{E}_{G}\left\{U_{\Pi}\left[\Pi_{0}^{*}\left(P^{f}, \tilde{Z}\right), \tilde{S}\right](1+\theta \tilde{Z}) \mid \tilde{S}\right\}, \mathrm{E}_{F}[\max (K-\tilde{P}, 0) \mid \tilde{S}]\right\}>(<) 0
$$

where $\Pi_{0}^{*}\left(P^{f}, Z\right)=(1+\theta Z)\left[P^{f} Q^{*}-C\left(Q^{*}\right)\right]+(1-\theta) Z$.

Proof. Suppose that $X(0)=Q^{*}$. In this case, the left-hand side of Eq. (18) becomes

$$
\begin{aligned}
& \int_{\underline{S}}^{\bar{S}} \mathrm{E}_{G}\left\{U_{\Pi}\left[\Pi_{0}^{*}\left(P^{f}, \tilde{Z}\right), S\right](1+\theta \tilde{Z}) \mid S\right\}\left[P^{f}-\mathrm{E}_{F}(\tilde{P} \mid S)\right] \mathrm{d} H(S) \\
& =-\operatorname{Cov}_{H}\left\{\mathrm{E}_{G}\left\{U_{\Pi}\left[\Pi_{0}^{*}\left(P^{f}, \tilde{Z}\right), \tilde{S}\right](1+\theta \tilde{Z}) \mid \tilde{S}\right\}, \mathrm{E}_{F}(\tilde{P} \mid \tilde{S})\right\},
\end{aligned}
$$

where the equality follows from Eq. (3) and the property of the covariance operator, $\operatorname{Cov}_{H}(\cdot, \cdot)$. Since $\mathrm{E}_{F}(\tilde{P} \mid S)$ is invariant to changes in $S$, the covariance term on the righthand side of Eq. (27) vanishes. Hence, $X(0)=Q^{*}$ is indeed the firm's optimal futures position when $Y=0$.

Substituting $X(0)=Q^{*}$ into the right-hand side of Eq. (19) yields

$$
\begin{aligned}
& \int_{\underline{S}}^{\bar{S}} \mathrm{E}_{G}\left\{U_{\Pi}\left[\Pi_{0}^{*}\left(P^{f}, \tilde{Z}\right), S\right](1+\theta \tilde{Z}) \mid S\right\}\left\{\mathrm{E}_{F}[\max (K-\tilde{P}, 0) \mid S]-\Phi\right\} \mathrm{d} H(S) \\
& =\operatorname{Cov}_{H}\left\{\mathrm{E}_{G}\left\{U_{\Pi}\left[\Pi_{0}^{*}\left(P^{f}, \tilde{Z}\right), \tilde{S}\right](1+\theta \tilde{Z}) \mid \tilde{S}\right\}, \mathrm{E}_{F}[\max (K-\tilde{P}, 0) \mid \tilde{S}]\right\}
\end{aligned}
$$

where the equality follows from Eq. (4) and the property of the covariance operator, $\operatorname{Cov}_{H}(\cdot, \cdot)$. Eqs. (9) and (28) and the second-order conditions for program (6) then imply that $Y^{*}>(<) 0$ if, and only if, condition (26) holds. 
The intuition for Proposition 4 is as follows. Since covariances can be interpreted as marginal variances, Eqs. (8) and (9) imply that the firm's optimal hedge position, $\left(X^{*}, Y^{*}\right)$, is the one that minimizes the variance of $U_{\Pi}[\Pi(\tilde{P}, \tilde{Z}), \tilde{S}](1+\theta \tilde{Z})$. Suppose that the firm chooses a full-hedge, i.e., $X=Q^{*}$, and uses no options, i.e., $Y=0$. In this case, the firm's profit at date 1 becomes $\Pi_{0}^{*}\left(P^{f}, \tilde{Z}\right)$. Since the expected price risk, $\mathrm{E}_{F}(\tilde{P} \mid S)$, is preserved as the realized state, $S$, varies, it follows from Eq. (2) that $\tilde{P}$ and $U_{\Pi}\left[\Pi_{0}^{*}\left(P^{f}, \tilde{Z}\right), \tilde{S}\right](1+\theta \tilde{Z})$ are state-independent. However, condition (26) ensures that $\max (K-\tilde{P}, 0)$ and $U_{\Pi}\left[\Pi_{0}^{*}\left(P^{f}, \tilde{Z}\right), \tilde{S}\right](1+\theta \tilde{Z})$ are positively (negatively) state-dependent. This implies that the firm can further reduce the variability of $U_{\Pi}\left[\Pi_{0}^{*}\left(P^{f}, \tilde{Z}\right), \tilde{S}\right](1+\theta \tilde{Z})$ by purchasing (selling) the put option contracts, thereby rendering the hedging role of options.

To gain more insight into the firm's optimal hedging decision, we consider the parametric example of Broll and Eckwert (1998) in which the firm's state-dependent utility function is given by $U(\Pi, S)=S \Pi-\gamma \Pi^{2} / 2$, where $\gamma$ is a positive constant such that $U_{\Pi}(\Pi, S)=$ $S-\gamma \Pi>0$ for all relevant values of $\Pi$. The firm's optimal output level, $Q^{*}$, solves $C^{\prime}\left(Q^{*}\right)=$ $P^{f}$. Suppose that the price risk, $\tilde{P}$, preserves its expected value as the realized state, $S$, varies so that $\mathrm{E}_{F}(\tilde{P} \mid S)=P^{f}$ for all $S \in[\underline{S}, \bar{S}]$. Suppose further that the background risk, $\tilde{Z}$, preserves its expected value and variance as $S$ varies so that $\mathrm{E}_{G}(\tilde{Z} \mid S)=0$ and $\mathrm{E}_{G}\left(\tilde{Z}^{2} \mid S\right)=\sigma_{Z}^{2}$ for all $S \in[\underline{S}, \bar{S}]$, where $\sigma_{Z}^{2}>0$ is the variance of $\tilde{Z}$. In this example, condition (26) reduces to $\operatorname{Cov}_{H}\left\{\tilde{S}, \mathrm{E}_{F}[\max (K-\tilde{P}, 0) \mid \tilde{S}]\right\}>(<) 0$. Solving the firm's optimal hedge position, $\left(X^{*}, Y^{*}\right)$, yields

$$
X^{*}=Q^{*}+\frac{\rho^{2} \operatorname{Cov}_{H}\left\{\tilde{S}, \mathrm{E}_{F}[\max (K-\tilde{P}, 0) \mid \tilde{S}]\right\}}{\gamma\left(1+\theta \sigma_{Z}^{2}\right)\left(1-\rho^{2}\right) \operatorname{Cov}[\tilde{P}, \max (K-\tilde{P}, 0)]},
$$

and

$$
Y^{*}=\frac{\operatorname{Cov}_{H}\left\{\tilde{S}, \mathrm{E}_{F}[\max (K-\tilde{P}, 0) \mid \tilde{S}]\right\}}{\gamma\left(1+\theta \sigma_{Z}^{2}\right)\left(1-\rho^{2}\right) \operatorname{Var}[\max (K-\tilde{P}, 0)]},
$$

where $\operatorname{Var}[\max (K-\tilde{P}, 0)]$ is the variance of $\max (K-\tilde{P}, 0), \operatorname{Cov}[\tilde{P}, \max (K-\tilde{P}, 0)]$ is the covariance between $\tilde{P}$ and $\max (K-\tilde{P}, 0)$, and $\rho \in(-1,0)$ is the correlation coefficient 
between $\tilde{P}$ and $\max (K-\tilde{P}, 0)$. It then follows from Eqs. (29) and (30) that $X^{*}<(>) Q^{*}$ and $Y^{*}>(<) 0$ if, and only if, $\operatorname{Cov}_{H}\left\{\tilde{S}, \mathrm{E}_{F}[\max (K-\tilde{P}, 0) \mid \tilde{S}]\right\}>(<) 0$, which are consistent with the results of Proposition $4 .^{11}$ When the state-dependent background risk is absence, i.e., $\tilde{Z} \equiv 0$, Eqs. (29) and (30) imply that $X^{*}$ is further away from $Q^{*}$ and $Y^{*}$ is further away from zero in this example when $\theta=1$.

\section{Conclusion}

In this paper, we examine the behavior of the competitive firm that possesses statedependent preferences and faces state-dependent price and background risk, where the background risk can be either additive or multiplicative. ${ }^{12}$ While the background risk is neither hedgeable nor insurable, the firm has access to fairly priced futures and option contracts to hedge against the price risk. We show that the separation theorem holds in that the firm's optimal output level depends neither on the risk attitude of the firm nor on the incident to the underlying uncertainty. We derive necessary and sufficient conditions under which banning the firm from hedging against the price risk has unambiguous effect on the firm's production decision. In contrast to the conventional wisdom, we construct a reasonable example to illustrate the plausible adverse effect of hedging on the firm's optimal output level. Finally, we derive necessary and sufficient conditions under which the full-hedging theorem holds and thus the firm does not use the option contracts for hedging purposes. When these conditions are violated, we show that the firm includes the option contracts in its optimal hedge position to better cope with the complicated statedependence structure between risk and preferences. This paper as such provides a rationale for the hedging role of options, which is over and above that of futures, in the case of

\footnotetext{
${ }^{11}$ Since $\max (K-P, 0)$ is convex in $P$, it follows from Rothschild and Stiglitz $(1971)$ that $\partial \mathrm{E}_{F}[\max (K-$ $\tilde{P}, 0) \mid S] / \partial S>(<) 0$ if an increase in the realized state, $S$, induces a mean-preserving-spread increase (decrease) in the price risk, $\tilde{P}$, in the sense of Rothschild and Stiglitz (1970). In this case, we have $\operatorname{Cov}_{H}\left\{\tilde{S}, \mathrm{E}_{F}[\max (K-\tilde{P}, 0) \mid \tilde{S}]\right\}>(<) 0$.

${ }^{12}$ Guo et al. (2015) have recently examined the competitive firm's production and hedging decisions when the firm is regret averse. It is of interest to extend their analysis by incorporating additive/multiplicative background risk. We leave this challenge for future research.
} 
state-dependent preferences and in the presence of state-dependent price and background risk.

\section{References}

Adam-Müller, A. F. A. (2000). Hedging price risk when real wealth matters. Journal of International Finance and Money, 19, 549-560.

Battermann, H. L., Braulke, M., Broll, U., \& Schimmelpfennig, J. (2000). The preferred hedge instrument. Economics Letters, 66, 85-91.

Briys, E., Crouhy, M., \& Schlesinger, H. (1993). Optimal hedging in a futures market with background noise and basis risk. European Economic Review, 37, 949-960.

Briys, E., \& Schlesinger, H. (1993). Optimal hedging when preferences are state dependent. Journal of Futures Markets, 13, 441-451.

Broll, U., \& Eckwert, B. (1998). Export and hedging decision with state-dependent utility. International Review of Economics and Finance, 7, 247-253.

Broll, U., \& Wong, K. P. (2002). Optimal full-hedging under state-dependent preferences. Quarterly Review of Economics and Finance, 42, 937-943.

Broll, U., \& Wong, K. P. (2015). The impact of inflation risk on forward trading and production. Portuguese Economic Journal, 14, 65-73.

Chavas, J.-P. (1985). On the theory of the competitive firm under uncertainty when initial wealth is random. Southern Economic Journal, 51, 818-827.

Fei, W., \& Schlesinger, H. (2008). Precautionary insurance demand with state-dependent background risk. Journal of Risk and Insurance, 75, 1-16.

Guo, X., Wong, W. K., Xu, Q. F., \& Zhu, X. H. (2015). Production and hedging decisions under regret aversion. Economic Modelling, 51, 153-158. 
Holthausen, D. M. (1979). Hedging and the competitive firm under price uncertainty. American Economic Review, 69, 989-995.

Karni, E. (1985). Decision making under uncertainty: The case of state-dependent preferences. Cambridge, MA: Harvard University Press.

Karni, E., Schmeidler, D., \& Vind, K. (1983). On state dependent preferences and subjective probabilities. Econometrica, 51, 1021-1031.

Kimball, M. S. (1990). Precautionary saving in the small and in the large. Econometrica, $58,53-73$.

Kimball, M. S. (1993). Standard risk aversion. Econometrica, 61, 589-611.

Lapan, H., Moschini, G., \& Hanson, S. D. (1991). Production, hedging, and speculative decisions with options and futures markets. American Journal of Agricultural Economics, $73,66-74$.

Machnes, Y. (1992). Production decisions in the presence of options. International Review of Economics and Finance, 1, 341-345.

Machnes, Y. (1993). Further results on comparative statics under uncertainty. European Journal of Political Economy, 9, 141-146.

Menezes, C. F., Geiss, C., \& Tressler, J. (1980). Increasing downside risk. American Economic Review, 70, 921-932.

Mossin, J. (1968). Aspects of rational insurance purchasing. Journal of Political Economy, $76,553-568$.

Rothschild, M., \& Stiglitz, J. E. (1970). Increasing risk I: A definition. Journal of Economic Theory, 2, 225-243.

Rothschild, M., \& Stiglitz, J. E. (1971). Increasing risk II: Its economic consequences. Journal of Economic Theory, 3, 66-84.

Sandmo, A. (1971). On the theory of the competitive firm under price uncertainty. Amer- 
ican Economic Review, 61, 65-73.

Wong, K. P. (1995). Further results on comparative statics under uncertainty: A comment on Machnes. European Journal of Political Economy, 11, 761-768.

Wong, K. P. (1996). Background risk and the theory of the competitive firm under uncertainty. Bulletin of Economic Research, 48, 241-251.

Wong, K. P. (2002). Production decisions in the presence of options: A note. International Review of Economics and Finance, 11, 17-25.

Wong, K. P. (2012a). Production and hedging under state-dependent preferences. Journal of Futures Markets, 32, 945-963.

Wong, K. P. (2012b). Production and futures hedging with state-dependent background risk. International Review of Economics and Finance, 24, 177-184.

Wong, K. P. (2013). International trade and hedging under joint price and exchange rate uncertainty. International Review of Economics and Finance, 27, 160-170.

Wong, K. P. (2014). Hedging and the competitive firm under correlated price and background risk. Decisions in Economics and Finance, 37, 329-340. 